\title{
Die Berliner Stiftung für Dermatologie in Zeiten des Umbruchs
}

\author{
The Berlin Foundation for Dermatology over the Years of Change
}

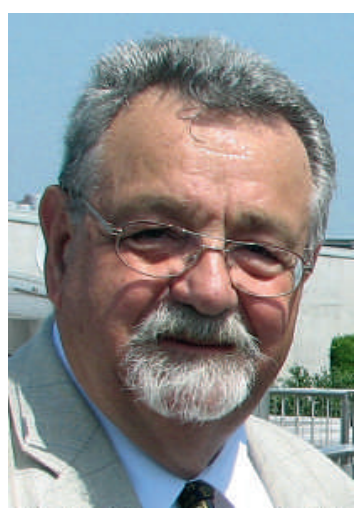

C. E. Orfanos

BSD-Geschäftsführer, Freie Universität Berlin
Bibliografie

Dol $10.1055 / \mathrm{s}-0028-1119615$ Akt Dermatol 2009; 35; 109 (c) Georg Thieme Verlag KG . Stuttgart · New York ISSN 0340-2541

Korrespondenzadresse

Prof. emer. Dr. Dr. h.c. Constantin E. Orfanos Sybelstr. 37 10629 Berlin

constantin.orfanos@charite.de
Die Dermato-Venerologie befindet sich im Umbruch. Das Fach, das nach den epochalen Entdeckungen des späten 19. Jahrhunderts schnell in eine Einheit zusammenwuchs, sich wissenschaftlich entwickeln und in der zweiten Hälfte des vergangenen 20. Jahrhunderts zu einer wichtigen medizinischen Disziplin reifen konnte, ist in einer Übergangsphase. Es ist heute offensichtlich auf der Suche nach einer neuen, überzeugenden Orientierung als Fundament für die Zukunft. Das gegenwärtige dermatologische Spektrum ist weit, außerordentlich vielfältig, und droht das Fach von innen her aufzubrechen. Es reicht von den Höhen einer supermodernen, ausgefeilten medizinischen Technologie über die klassische klinische Dermatologie, einschließlich der klinisch-experimentellen Forschung, bis in die flache Routine und manche Niederungen eines Kosmetikgeschäfts. In dieser vielgesichtigen Breite liegt ein Risiko; es ist schwer zu sagen, wie weit das Auseinanderdriften reichen wird, und mit welchen Folgen.

Dazu kommt, dass wenige andere Fächer der Medizin so weit und nachhaltig von den sozial-ökonomischen Problemen der Globalisierung und den Notwendigkeiten gefordert werden, die sich heute in der medizinischen Versorgung der Menschen in der Dritten Welt offenbaren. Denn, mit der Bekämpfung (a) der HIV-Infektion und ihrer verheerenden Folgen, (b) der weit verbreiteten sexuell übertragbaren Infektionen, und schließlich auch (c) der in vielen Ländern immer noch vorhandenen Lepra, gehören drei der bedeutendsten Zielsetzungen der Weltgesundheitsorganisation WHO zu den Interessen- und Aufgabengebieten der Dermatologie. Hier aber, zwischen der praktischen Ausübung des Faches in den reichen Ländern des Westens und den Bedürfnissen and Zwängen einer adäquaten dermatologischen Versorgung in den Ländern Afrikas entstehen gewaltige Spannungsfelder, die schwer zu überbrücken sind.

Dabei scheint sich die westliche Medizin mit ihrer naturwissenschaftlichen Orientierung etwas übernommen zu haben; die ärztliche Heilkunst bleibt vielerorts auf der Strecke, ökonomische Engpässe bringen neue Probleme mit sich und potenzieren die Gefahren, die sich aus einem sehr eng gehaltenen naturwissenschaftlichen Blickwinkel für die ethischen Grundlagen des Arztberufes ergeben. Hier liegen weitere Risiken. Mit dem Einzug der Ökonomie in die Medizin werden durch betriebswirtschaftliche Überlegungen im Ablauf der klinischen Routine am Krankenbett die hohen ethischen Anforderungen an den Arztberuf zumindest unterminiert, wenn nicht in Frage gestellt, und durch eine monetäre Sichtweise des ärztlichen Berufes ersetzt. Bedauerlich ist, dass Bildung und Gesundheit trotz aller Beteuerungen in der Öffentlichkeit keine hohe politische Priorität eingeräumt wird; der Staat zieht sich von seiner Verantwortung merklich zurück, das Gesundheitssystem bzw. seine Institutionen bleiben unterfinanziert. Es ist allseits modern geworden, auf die Notwendigkeit von Drittmitteleinwerbungen hinzuweisen, wobei die aktuelle internationale Finanzkrise den Bedarf noch verschlimmert.

In diesen Zeiten des Umbruchs ist die Gründung und Tätigkeit von Stiftungen von immer größer werdender Bedeutung, auch wenn Stiftungen von der Finanzhöhe her bei weitem nicht genügen, um die anstehenden Probleme zu lösen. Von der ideellen Seite und ihrer Bedeutung her sind sie aber für jedes Fach ein großer Gewinn. Die Aufgaben, die sich als Stiftungsziele eignen, sind in der Dermatologie überall und leicht zu greifen, sie werden gerade in unserem Fach besonders gut wahrnehmbar, dessen sozioökonomische Bedeutung immer ein wichtiger Teil war. Man darf es nicht vergessen, es waren gerade die sog. Geschlechtskrankheiten, die im 19. Jahrhundert die Geburtsstunde der Dermato-Venerologie einläuteten.

Die Berliner Stiftung für Dermatologie (BSD) wurde innerhalb der Freien Universität Berlin in den 90er-Jahren nach mehrjährigen Bemühungen der zwei Stifter gegründet. Ihr Anliegen war es, erwirtschaftete Erträge aus dem in Berlin organisierten und mit überwältigendem Erfolg abgelaufenen 17. Weltkongresses für Dermatologie 1987 finanztechnisch in eine für die Deutsche Dermatologie sinnvolle Aufgabe zu investieren. Es war der damalige Kongresspräsident Günter Stüttgen, der sich mit aller Kraft dafür eingesetzt hat. Heute blickt die Berliner Stiftung auf ein Jahrzehnt erfolgreicher Stiftungsarbeit zurück. 10 Hauptpreisträger und über 30 Stipendiaten und andere Wissenschaftler aus 20 verschiedenen Ländern, die von der Stiftung bisher in unterschiedlicher Weise finanziell unterstützt wurden, sprechen eine klare Sprache.

Während zu Beginn ausschließlich Fortbildungsund Forschungsaspekte im Vordergrund standen, ist heute die Förderung der dermatologischen Weiterbildung von jüngeren Kollegen und die Versorgung von Patienten, zumal in den Ländern Afrikas, wichtiger Teil der Zielsetzungen der BSD. Eine Reihe von Universitäts- und Städtischen Hautkliniken bzw. Instituten aus mehreren Bun- 
desländern haben dabei dankenswerterweise der Stiftung in der Erfüllung ihrer Ziele geholfen.

Dem 10-jährigen Jubiläum der Berliner Stiftung und in Anerkennung der bisher geleisteten Stiftungsarbeit ist dieses Heft der „Aktuellen Dermatologie“ gewidmet. Am 23. Mai 2009 findet ein Symposion in bemessenem Rahmen in Berlin statt, wozu jeder interessierte Kollege gern und kostenlos teilnehmen kann.

Im vorliegenden Heft findet der Leser eine Zahl interessanter Beiträge prominenter Vertreter des Faches und kundiger Autoren, die die Aktualität der gewählten Thematiken klar vor Augen führen. Neben vier wichtigen klinischen Fragestellungen, wie die Allergien, die kutanen Lymphome, das maligne Melanom und die atypischen Mykobakteriosen, sind auch Aufsätze über hochaktuelle berufs- und universitätspolitische Fragestellungen zu lesen: Dazu gehören der Stand der überaus notwendigen Harmonisierung der dermatologischen Aus- und Weiterbildung in den Ländern des Vereinigten Europas, die Möglichkeit eines sog. „outsourcing“, um die akademische Lehre auch unter schwierigen Umständen zu gewährleisten, sowie Gedanken über medizinisch-ethische Konfliktfelder, die neuerdings durch den Einzug der Ökonomie und Hochtechnologie in der Medizin aufkommen und den praktizierenden Arzt belasten. Schließlich, als Hinweis auf die Zukunft, findet sich in diesem Heft eine umfassende Übersichtsarbeit über die Stammzellpopulationen, die sich aus der Haut und ihrer Anhangsorgane in vitro gewinnen lassen, und die Möglichkeiten, die sich damit für die medizinische Forschung abzeichnen.

Das Kuratorium der BSD ist Frau Prof. Bayerl und dem gesamten Herausgeber-Kollegium der „Aktuellen Dermatologie“ sehr verbunden dafür, dass dieses Heft in der vorliegenden Form anlässlich des 10-jährigen Jubiläums der Stiftung erscheint. Für alle Beiträge zur Realisierung der genannten Stiftungsziele ist das Kuratorium dankbar, über die vielen Möglichkeiten dies zu tun, erteilt der Autor gern Auskunft. Jede weitere Förderung der Berliner Stiftung für Dermatologie ist ein Gewinn für unser Fach. 